\title{
Avaliação do estado nutricional de crianças e adolescentes na admissão em uma unidade de terapia intensiva pediátrica
}

\author{
Nutritional assessment of children and adolescents on admission to \\ a pediatric intensive care unit
}

\author{
Letícia Dione Caruccioa, Vera Lúcia Bosab, Tatiana Maraschinc, \\ Paulo Roberto Antonacci Carvalho ${ }^{\mathrm{d}}$ \\ a Nutricionista. Especialista em Saúde da Criança pelo Programa de Residência Multiprofissional do Hospital de Clínicas de Porto Alegre (HCPA), \\ Porto Alegre, RS, Brasil. \\ Nutricionista. Doutora pelo Programa de Pós graduação em Saúde da Criança e do Adolescente. Professora Adjunta do Departamento de Nutrição \\ da Universidade Federal do Rio Grande do Sul (UFRGS), Porto Alegre, RS, Brasil. \\ c Nutricionista. Especialista em Nutrição Clínica e Gestão Hospitalar, HCPA, Porto Alegre, RS, Brasil. \\ dMédico. Doutor em Ciências Médicas: Pediatria. Professor Titular do Departamento de Pediatria da Faculdade de Medicina da UFRGS, \\ Porto Alegre, RS, Brasil. \\ Financiamento: $\mathrm{O}$ estudo recebeu financiamento da Fundação de Incentivo à Pesquisa (FIPE)/Brasil.
}

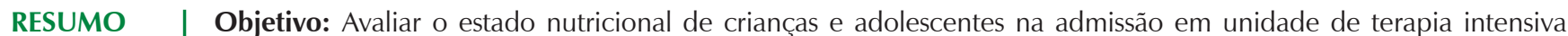
pediátrica de um hospital público de referência.

Materiais e Métodos: Estudo transversal que avaliou 277 pacientes, de 0 a 19 anos que foram admitidos na unidade de terapia intensiva pediátrica, nas primeiras $72 \mathrm{~h}$ de internação, no ano de 2013. As variáveis avaliadas, a partir dos prontuários, foram as medidas antropométricas de peso e estatura. Para verificar a associação do estado nutricional com as demais variáveis, utilizou-se o teste qui-quadrado, sendo considerado significativo $p<0,05$.

Resultados: Entre os 277 pacientes, 53,4\% $(n=148)$ eram do sexo masculino. Encontrou-se 10,5\% $(n=21)$ de desnutrição, 20,1\% $(n=40)$ de excesso de peso e $69,34 \%(n=138)$ de eutrofia. Destaca-se, que a desnutrição foi mais prevalente entre os menores de 2 anos, enquanto o excesso de peso foi maior na faixa etária de 2 a 10 anos. Em 28,79\% ( $n=57)$ da amostra foi encontrado baixa estatura, com maior prevalência entre os menores de 2 anos $(p<0,001)$, podendo estar associado ao elevado número de prematuros na amostra $(n=26, p=0,003)$.

Conclusão: Na admissão de crianças e adolescentes internados em unidade de terapia intensiva pediátrica, desnutrição foi o estado nutricional menos prevalente quando comparamos com outros estudos. O número expressivo de prematuros pode estar associado à alta prevalência de baixa estatura encontrada.

Palavras-chave: avaliação nutricional; pediatria; paciente crítico; unidade terapia intensiva; prematuridade.

ABSTRACT

Objective: To evaluate the nutritional status of children and adolescents on admission in the pediatric intensive care unit in a public referral hospital.

Material and Methods: Cross-sectional study that evaluated 277 patients aged 0 to 19 years who were admitted to the pediatric intensive care unit in the first 72 hours of hospitalization in the year 2013. The variables, from medical records, were the anthropometric measurements of weight and height. To investigate the association between nutritional status and other variables, the chi-squared test was used, considering a significance level of $p<0.05$.

Results: Of the 277 patients, $53.4 \%(n=148)$ were male and $10.5 \%$ were classified as $(n=21)$ malnutrition, $20.1 \%(n=40)$ as overweight and $69.34 \%(n=138)$ as eutrophic. Malnutrition was more prevalent among children younger than 2 years, while overweight was higher in the age group of 2 to 10 years. In $28.79 \%(n=57)$ of the sample short stature was found, with higher prevalence among children under 2 years $(p<0.001)$ and may be associated with the high number of premature infants in the sample $(n=26, p=0.003)$.

Conclusion: In the admission of children and adolescents in pediatric intensive care unit, malnutrition was the least prevalent nutritional status when compared to other studies. The significant number of premature infants may be associated with high prevalence of short stature found.

Keywords: nutritional assessment; pediatrics; critically ill; intensive care unit; prematurity. 


\section{INTRODUÇÃO}

Desnutrição tem sido relatada, na admissão e durante hospitalização, em crianças criticamente doentes desde os anos $80^{1}$. Nilesh et al. ${ }^{2}$, em um estudo de coorte multicêntrico realizado em 31 Unidades de Terapia Intensiva Pediátrica (UTIP) de oito países, observou que mais de $30 \%$ dos pacientes apresentavam desnutrição na sua admissão. Em UTIP, crianças com desnutrição ficam maior tempo em ventilação mecânica, apresentam maior tempo de internação e maior mortalidade. Crianças criticamente doentes frequentemente tem baixas reservas nutricionais e estão em maior risco para desnutrição ${ }^{3}$. Condições como doença cardíaca congênita, doenças oncológicas, disfunção neurológica significativa e outras condições crônicas comuns, contribuem para o risco nutricional' 1 .

Realizar triagem do estado nutricional, prévia, em pacientes críticos ajuda a identificar pacientes em risco nutricional e facilita o início da terapia nutricional ${ }^{4}$. Esta triagem ou avaliação nutricional inicial inclui vários componentes: história médica, história dietética, dados bioquímicos e medidas antropométricas. Medidas antropométricas incluem Peso (P), Comprimento/Estatura (E), Prega Cutânea Tricipital (PCT), e Circunferência muscular do braço $(\mathrm{CMB})$. São medidas simples, reprodutíveis e de baixo custo; contudo podem ser influenciadas por edema. Estas simples medidas habilitam o cálculo do Índice de Massa Corporal (IMC), razão do Peso/Estatura (P/E), razão Estatura/Idade (E/I) e razão Peso/Idade $(\mathrm{P} / \mathrm{l})^{5}$. A avaliação nutricional completa torna-se muito importante em pacientes pediátricos, pois crianças podem desnutrir mais rapidamente, quando comparamos com adultos ${ }^{6}$. A aferição do peso e da estatura durante a admissão na UTIP é sempre indicada, e segundo Verger et al. ${ }^{1}$, para uma criança hospitalizada o crescimento tem importância vital, e a perda de peso, é o melhor exame físico preditor de risco nutricional ${ }^{7}$.

Em geral, o desenvolvimento ou permanência de um estado de desnutrição durante hospitalização em UTIP é devido à doença de base, condições nutricionais e suporte nutricional inadequado. Nestes pacientes, desnutrição é associada com instabilidade fisiológica. Como resultado, maior cuidado clínico intensivo é necessário, e a prevalência de mortalidade é elevada ${ }^{8}$.

Diante deste enfoque, e considerando a importância das condições nutricionais para um bom prognóstico de crianças e adolescentes hospitalizados, especialmente aqueles criticamente doentes, este trabalho propõem-se a identificar o estado nutricional de população pediátrica na admissão em uma Unidade de Terapia Intensiva.

\section{MATERIAIS E MÉTODOS}

Estudo de caráter quantitativo e descritivo, com corte transversal, foi conduzido na UTIP do Hospital de Clínicas de Porto Alegre - Hospital Terciário de Alta Complexidade, localizado em Porto Alegre, Rio Grande do Sul, e que atende cerca de 50 pacientes por mês, provenientes de Porto Alegre e interior do Estado, que abrange uma população em torno de 10 milhões de habitantes.

O estudo avaliou um total de 277 pacientes internados em 2013. A coleta ocorreu entre abril e julho de 2015. Os dados foram coletados diretamente dos prontuários dos pacientes selecionados por técnica de amostragem aleatória simples. A busca dos prontuários e dos dados envolvidos na pesquisa foi acessada através do Serviço de Arquivo Médico e Informações em Saúde - SAMIS - do Hospital de Clínicas de Porto Alegre. A partir dos dados coletados dos prontuários foram calculados os índices antropométricos para definir o diagnóstico nutricional.

A população estudada foi composta por pacientes de ambos os sexos, de 0 a 19 anos que foram admitidos na UTIP no ano de 2013. Foram excluídos pacientes que apresentavam Nanismo, Paralisia Cerebral e Síndrome de Down. O cálculo do tamanho da amostra foi realizado com base no estudo de Goh et al. ${ }^{9}$, que realizou avaliação nutricional de 1146 crianças na admissão em Unidade de Terapia Intensiva de Hospital Pediátrico. Para nível de confiança de $95 \%$, proporção de desnutrição estimada em $10 \%$, margem de erro de $3 \%$ e população estimada em 650 pacientes, obtiveram-se um total mínimo de 242 pacientes. Para o cálculo foi utilizado o software WinPepi versão 11.43.

O trabalho seguiu a seguinte metodologia em relação à coleta dos dados, instrumentos, métodos e referências: Os pacientes foram pesados e medidos no primeiro dia de internação. Foi considerada a avaliação realizada nas primeiras $72 \mathrm{~h}$ de internação na UTIP, seguindo protocolo de medidas do serviço.

As variáveis avaliadas a partir dos prontuários foram peso e estatura. A partir das medidas antropométricas foram calculados no software Anthro e Anthro Plus, os índices antropométricos de: Peso para Estatura (P/E), Estatura para Idade (E/I) e Índice de Massa Corporal para Idade (IMC/I). Os dados clínico-demográficos avaliados foram: sexo, idade na data de admissão e doença de base. Para o diagnóstico nutricional, utilizou-se os valores críticos de escore $z(<-3)$ para muito baixo peso, $(\geq-3$ e $<-2)$ para baixo peso, $(\geq-2$ e $\leq+2)$ para eutrofia e $(>+2)$ para excesso de peso, a partir dos índices antropométricos de Peso/Estatura (P/E) para crianças até 2 anos, Índice de Massa Corporal para Idade 
(IMC/I) para crianças maiores de 2 anos. O índice Estatura/ Idade (E/I) foi utilizado para todas as faixas etárias, conforme referência das curvas de crescimento da Organização Mundial da Saúde ${ }^{10}$ e os valores críticos de z escore foram os seguintes: $(<-3)$ para muito baixa estatura, $(\geq-3$ e $<-2)$ para baixa estatura e $(\geq-2)$ para estatura adequada para idade. Para avaliação dos pré termos corrigiu-se a idade cronológica de acordo com a idade gestacional ${ }^{11}$.

As crianças com estado nutricional de baixo peso, risco nutricional e desnutrição foram agrupadas no estado nutricional de desnutrição e crianças com diagnóstico nutricional de risco para sobrepeso, sobrepeso, obesidade e obesidade grave foram agrupadas no estado nutricional de excesso de peso. Também foi pesquisada nos prontuários de cada paciente, a doença de base, sendo categorizadas em: prematuridade e suas comorbidades, doenças crônicas (doenças hepáticas, cardíacas, renais, neurológicas e respiratórias como p.ex. a asma), doenças neoplásicas, síndromes ou malformações (erros inatos do metabolismo, epilepsia, doenças genéticas) e doenças ou situações agudas (atropelamento, acidentes, leptospirose, sepse, bronquiolites, etc). As informações obtidas foram armazenadas em um banco de dados elaborado no Statiscal Package for Social Sciences (SPSS) versão 18.0 com dupla digitação e posterior validação, e as análises foram efetuadas no mesmo programa. Foi realizada estatística descritiva, sendo as variáveis categóricas apresentadas em frequência absoluta e percentual. Para verificar a associação do estado nutricional com as demais variáveis (peso, prematuridade, idade, sexo e doença de base) utilizou-se o teste qui quadrado, sendo considerado significativo $p<0,05$. As questões éticas do presente estudo seguem as Diretrizes e Normas Reguladoras de Pesquisas envolvendo Seres Humanos (Resolução 466/2012). Este projeto faz parte de um trabalho maior intitulado Comparação entre Nomograma e Fórmula Simplificada no Cálculo da Superfície Corporal em Crianças Desnutridas e Bem Nutridas em UTI Pediátrica de Hospital Terciário, submetido à avaliação do Grupo de Pesquisa e Pós Graduação (GPPG) e Comitê de Ética do HCPA e aprovado sob protocolo número 110466.

\section{RESULTADOS}

O estudo avaliou um total de 277 pacientes, dos quais $53,4 \%(n=148)$ eram do sexo masculino. A Tabela 1 apresenta a característica geral da amostra. Importante salientar que da amostra total, $27,8 \%(n=77)$ não tiveram comprimento aferido, assim como $16,97 \%(n=47)$ não tiveram peso avaliado no período de internação, que consideramos como as primeiras $72 \mathrm{~h}$ de admissão na unidade, sendo que estes foram excluídos da amostra válida que compreendeu
199 crianças e adolescentes. A faixa etária mais prevalente foi de 0 a 2 anos, que compreendeu $58,5 \%(n=162)$ da amostra total. Dentre as doenças de base mais prevalentes encontraram-se doenças relacionadas a síndromes e malformações, doenças agudas e doenças oncológicas.

Tabela 1. Características clínicas e demográficas de crianças e adolescentes na admissão em unidade de terapia intensiva pediátrica.

\begin{tabular}{lc}
\hline Características & $\mathbf{n}(\%)$ \\
Idade & $162(58,5)$ \\
$0-2$ anos & $70(83,8)$ \\
$2-10$ anos & $45(16,2)$ \\
$>10$ anos & $277(100)$ \\
Total & \\
Sexo & $148(53,4)$ \\
Masculino & $129(46,6)$ \\
Feminino & $277(100)$ \\
Total & \\
Doença de Base & $31(11,2)$ \\
Prematuridade e complicações & $45(16,2)$ \\
Doenças crônicas* & $50(18,1)$ \\
Doenças oncológicas & $82(29,6)$ \\
Síndromes/malformações & $61(22,0)$ \\
Doenças agudas & $8(2,9)$ \\
Outros & $277(100)$ \\
\hline Total & \\
\hline * Doenças crônicas (cardíacas, hepáticas, renais, neurológicas, oncológicas e respira- \\
tórias).
\end{tabular}

Em relação ao estado nutricional da amostra final, conforme a Figura 1 demonstra, encontramos diagnóstico nutricional de desnutrição em 10,5\% $(n=21)$, excesso de peso em 20,1\% ( $n=40)$, e maioria eutrófica em $69,34 \%$ $(n=138)$, ou seja, bom estado nutricional. Em relação à estatura foi possível observar que $28,79 \%(n=57)$ da amostra apresentava baixa estatura. A Tabela 2 mostra os indicadores nutricionais por faixa etária, e pode-se verificar que na faixa etária de 0-2 anos, conforme o indicador antropométrico de $P / E, 13,5 \%(n=15)$ das crianças estavam desnutridas, sendo a maioria apresentando-se com estado nutricional adequado. A Tabela 2 também mostra que naqueles grupos com idade acima de 2 anos, a maioria apresentava estado nutricional adequado, com valores estatisticamente relevantes, sendo a maior prevalência de eutrofia encontrada naqueles maiores de 10 anos. Quando comparamos desnutrição entre todas as faixas etárias, podemos verificar que este estado nutricional foi mais prevalente entre os menores de 2 anos (independente do índice antropométrico) e excesso de peso mais prevalente na faixa etária entre 2-10 anos, 24\% $(n=14)$. 


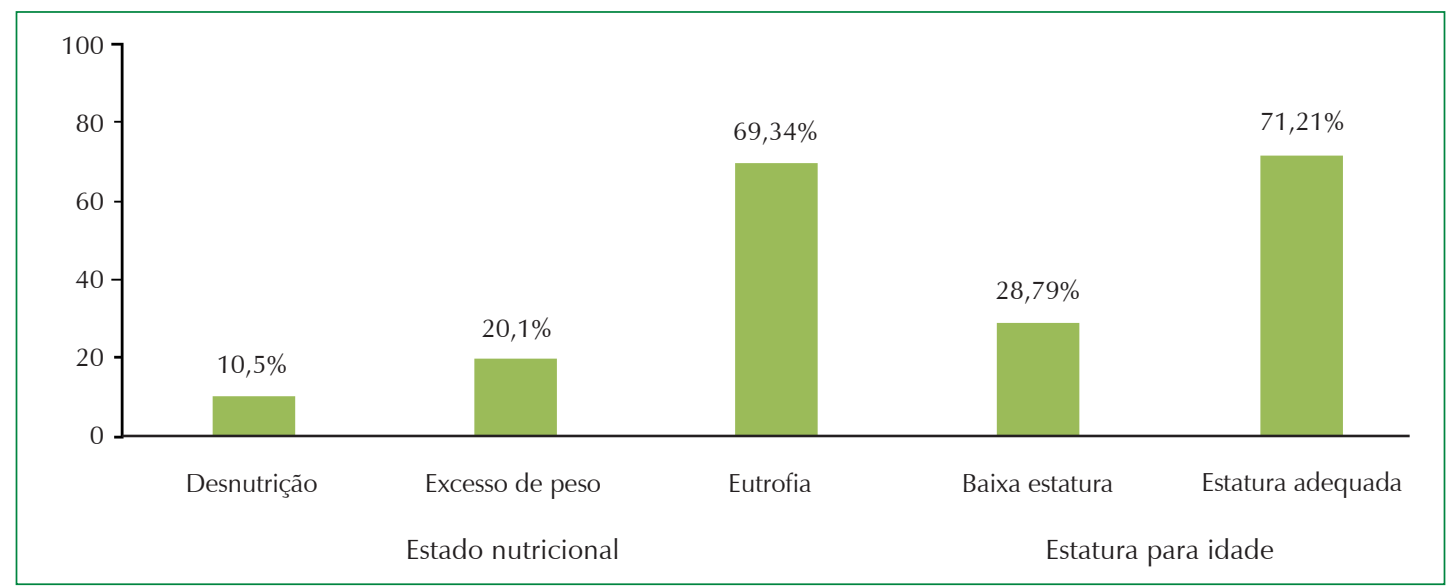

Figura 1. Classificação do estado nutricional de crianças e adolescentes na admissão em unidade de terapia intensiva pediátrica. ( $n=199$ - amostra válida).

Tabela 2. Indicadores nutricionais por faixa etária de crianças e adolescentes na admissão em unidade de terapia intensiva pediátrica.

\begin{tabular}{|c|c|c|c|c|}
\hline \multirow{3}{*}{ Indicadores } & \multicolumn{3}{|c|}{ Faixa etária } & \multirow{3}{*}{$p^{*}$} \\
\hline & 0-2 anos) & $2-10$ anos & $>10$ anos & \\
\hline & n (\%) & n (\%) & n (\%) & \\
\hline IMC para idade & & & & 0,014 \\
\hline Magreza acentuada & $13(11)$ & $2(3,5)$ & $0(0)$ & \\
\hline Magreza & $13(11)$ & $3(5,3$ & $1(3,6)$ & \\
\hline Eutrofia & $76(67)$ & $38(67$ & $22(79)$ & \\
\hline Risco ou Sobrepeso & $11(9,6)$ & $7(12)$ & $3(11)$ & \\
\hline Obesidade & $1(0,9)$ & $7(12)$ & $2(7,1)$ & \\
\hline Total & $114(100)$ & $57(100)$ & $28(100)$ & \\
\hline Peso para estatura & & & & 0,934 \\
\hline Magreza Acentuada & $8(7,2)$ & $1(1,6)$ & --- & \\
\hline Magreza & $7(6,3)$ & $5(8,1)$ & & \\
\hline Eutrofia & $75(68)$ & $47(76)$ & & \\
\hline Risco de Sobrepeso & $16(14)$ & $6(9,7)$ & & \\
\hline Sobrepeso & $4(3,6)$ & $2(3,2)$ & & \\
\hline Obesidade & $1(09)$ & $1(1,6)$ & & \\
\hline Total & $111(100)$ & $62(100)$ & & \\
\hline Estatura para idade & & & & $<0,001$ \\
\hline Muito baixa estatura para idade & $28(25)$ & $5(8,8)$ & $0(0)$ & \\
\hline Baixa estatura para idade & $21(18)$ & $3(5,3)$ & $0(0)$ & \\
\hline Estatura adequada para idade & $65(57)$ & $49(86)$ & $27(100)$ & \\
\hline Total & $114(100)$ & $57(100)$ & $27(100)$ & \\
\hline
\end{tabular}

* Teste qui-quadrado, $p<0,05$.

Os resultados foram estatisticamente significativos quando relacionamos faixa etária com o índice antropométrico de $E / I(p<0,001)$, onde observamos um déficit de estatura em $43 \%(n=49)$ da amostra de crianças menores de dois anos de idade, e $14,1 \%(n=8)$ naqueles com idades entre 2 e 10 anos. Encontrou-se estatura adequada entre todos pacientes maiores de 10 anos. Quando relacionamos o diagnóstico nutricional (utilizando o $\mathrm{P} / \mathrm{E}$ até 2 anos e IMC/I para os maiores de 2 anos) com todas as variáveis (Tabela 3), foi encontrada diferença significativa com a variável sexo, demonstrando que os meninos apresentaram maior prevalência de excesso de peso, enquanto que as meninas são mais desnutridas. 
Tabela 3. Associação do diagnóstico nutricional com as variáveis clínicas demográficas e nutricionais de crianças e adolescentes na admissão em unidade de terapia intensiva pediátrica.

\begin{tabular}{|c|c|c|c|c|}
\hline \multirow{3}{*}{ Variáveis } & \multicolumn{3}{|c|}{ Diagnóstico Nutricional (P/E $<2$ anos, IMC $>2$ anos) } & \multirow{3}{*}{$p^{*}$} \\
\hline & $\begin{array}{l}\text { Magreza } \\
(n=21)\end{array}$ & $\begin{array}{l}\text { Eutrofia } \\
(n=138)\end{array}$ & $\begin{array}{l}\text { Excesso de peso } \\
\qquad(n=40)\end{array}$ & \\
\hline & n (\%) & n (\%) & $n(\%)$ & \\
\hline Sexo & & & & 0,007 \\
\hline Masculino & $6(28,60)$ & $71(51,40)$ & $28(70,00)$ & \\
\hline Feminino & $15(71,40)$ & $67(48,60)$ & $12(30,00)$ & \\
\hline Idade & & & & 0,492 \\
\hline $0-2$ anos & $15(71,4)$ & $78(56,50)$ & $21(52,50)$ & \\
\hline 2-10 anos & $5(23,80)$ & $38(27,5)$ & $14(35,00)$ & \\
\hline$>10$ anos & $1(4,8)$ & $22(15,90)$ & $5(12,50)$ & \\
\hline Total & $21(100)$ & $138(100)$ & $40(100)$ & \\
\hline Estatura para idade & & & & 0,529 \\
\hline Muito baixa estatura para idade & $3(14,30)$ & $21(15,20)$ & $9(23,10)$ & \\
\hline Baixa estatura para idade & $3(14,30)$ & $19(13,80)$ & $2(5,10)$ & \\
\hline Estatura adequada para idade & $15(71,40)$ & $98(71,00)$ & $28(71,80)$ & \\
\hline Total & $21(100)$ & $138(100)$ & $39(100)$ & \\
\hline Doença de Base & & & & 0,271 \\
\hline Prematuridade e complicações & $0(0,00)$ & $14(10,10)$ & $5(12,50)$ & \\
\hline Doenças crônicas & $4(19)$ & $26(18,80)$ & $5(12,50)$ & \\
\hline Doenças oncológicas & $7(33,3)$ & $32(23,20)$ & $6(15,00)$ & \\
\hline Síndromes/malformações & $8(38,1)$ & $40(29,00)$ & $10(25,00)$ & \\
\hline Doenças agudas & $2(9,50)$ & $24(17,40)$ & $12(30,00)$ & \\
\hline Outros & $0(0,00)$ & $2(1,40)$ & $2(5,00)$ & \\
\hline Total & $21(100)$ & $138(100)$ & $40(100)$ & \\
\hline
\end{tabular}

* Teste qui-quadrado.

Para as demais variáveis não se observou diferenças significativas, porém é importante destacar que entre os eutróficos, 29\% $(n=40)$ apresentaram déficit de estatura.

Relacionando estado nutricional com doença de base, verifica-se que a maioria dos desnutridos apresentava síndromes/malformações ou doenças oncológicas (Tabela 3). Entre aqueles com excesso de peso, a maioria apresentava doença aguda. Cabe destacar que os prematuros $(n=26)$ foram aqueles que apresentaram maior prevalência $(53,9 \%)$ de baixa estatura quando comparados com os nascidos a termo $(p=0,003)$ - dados não apresentados.

\section{DISCUSSÃO}

O presente estudo avaliou o estado nutricional dos pacientes pediátricos na UTIP do Hospital de Clínicas de Porto Alegre, hospital terciário de alta complexidade, localizado na Região Sul do Brasil. Considerando a amostra válida, encontrou-se praticamente a mesma prevalência de desnutrição $(10,5 \%)$ que o estudo de Goh et al. ${ }^{9}$, que avaliou 1146 pacientes ( 2 a 18 anos), também no período de admissão em uma UTIP. O mesmo mostrou 10,1\% de desnutrição e $65,7 \%$ de bom estado nutricional, também de acordo com a classificação de escore $z^{9}$. A maioria dos estudos sobre avaliação nutricional em UTIP, nas últimas décadas, tem mostrado prevalência de desnutrição maior do que foi demonstrada no presente trabalho, variando entre $24 \%$ e $55 \%{ }^{12}$. Outro estudo, também retrospectivo, realizado no México, que avaliou estado nutricional de crianças e adolescentes na admissão em UTIP, encontrou 36,2\% de desnutrição ${ }^{13}$. No Brasil, estudo de coorte prospectivo realizado em Hospital Universitário do Estado de São Paulo constatou que $45 \%$ dos pacientes eram desnutridos no momento da admissão na UTIP. Cabe destacar que a alta prevalência de desnutridos, segundo os próprios autores, seria explicada pelo elevado número de pacientes $(84,7 \%)$ que foram diagnosticados com doença crônica pré-existente ${ }^{14}$.

Crianças que são admitidas em hospital, principalmente em unidades de terapia intensiva, apresentam um risco elevado para desenvolver desnutrição, principalmente crianças com doença de base e condições clínicas graves ${ }^{15}$. 
Entre as principais doenças de base encontradas no presente estudo estão as síndromes e malformações, doenças ou situações agudas, considerando que estas eram crianças e adolescentes previamente hígidos, e doenças oncológicas. Estes dados mostram um perfil de doenças diferenciado de outras unidades de tratamento intensivo pediátrico. No trabalho de Menezes et al. ${ }^{14}$, as doenças pré-existentes ou crônicas (doenças do coração, renais e hepáticas) foram mais prevalentes, e quando associado à desnutrição, não foi demonstrado nenhum efeito sobre o tempo de internação na UTIP.

A maior parte da população estudada foi composta por crianças menores de 2 anos, e conforme o índice de Peso para Estatura, grande parte delas apresentaram baixa estatura para idade atual. Pode-se relacionar este dado com o número expressivo de prematuros identificados neste grupo. O crescimento é considerado um processo contínuo e complexo, resultante da interação de fatores genéticos, nutricionais, hormonais e ambientais. Os recém-nascidos prematuros possuem um padrão próprio de crescimento e desenvolvimento, que varia de dias a semanas, em função da idade gestacional, do peso ao nascer e do tempo de duração de determinada doença ${ }^{16}$. Recente trabalho, que avaliou crianças nesta mesma faixa etária (menores de dois anos) na admissão hospitalar, envolvendo 10 hospitais universitários, de várias regiões do Brasil, também demonstrou uma elevada prevalência de baixa estatura, em torno de $30 \%{ }^{17}$.

A população geral de crianças, na Região Sul do Brasil, também apresenta déficit de estatura. No estudo realizado sobre cobertura do Sistema de Vigilância Alimentar e Nutricional (SISVAN) em crianças do Rio Grande do Sul foi encontrado $9,1 \%$ de déficit de estatura entre crianças de 0 a 5 anos $^{18}$. A avaliação da estatura e do crescimento é, sumariamente, a melhor medida de avaliação para desnutrição crônica, sendo que déficit linear reflete exposição à um ambiente deficiente, isto é, dieta e cuidado inadequados, além de doença ${ }^{19}$.

Em relação à obesidade infantil, estudos referem que a prevalência na população geral na faixa etária de 2 a 18 anos nos EUA é de $17 \%$, e a obesidade entre pacientes críticos pediátricos é de aproximadamente $13 \%{ }^{19}$. Neste trabalho encontrou-se prevalência de excesso de peso maior na faixa etária entre 2 e 10 anos, sendo excesso de peso englobando estado nutricional de risco para sobrepeso, sobrepeso, obesidade e obesidade grave. Também foi verificado que entre os meninos houve prevalência maior de excesso de peso. Araújo et al. ${ }^{20}$, quando estudou o estado nutricional de adolescentes que fazem parte de um estudo de coorte na cidade de Pelotas, encontrou maior prevalência de obesidade entre meninos. Estes dados refletem também o estado nutricional da população geral, quando observamos os resultados da Pesquisa Nacional de Saúde Escolar (PENSE) desenvolvido em $2009^{21}$. Este que foi o primeiro estudo nacional em amostra representativa de escolares no conjunto das capitais brasileiras a estimar a prevalência de fatores de risco e de proteção à saúde que envolveu estudantes do ensino fundamental de escolas públicas e privadas. $\mathrm{O}$ excesso de peso foi o principal problema nutricional verificado entre estes escolares de todas as capitais e Distrito Federal do país, estudados pela PENSE. Como a prevalência da obesidade é elevada e por causa de sua frequente associação com muitas doenças, a prevalência de obesidade entre crianças hospitalizadas é concomitantemente elevada em aproximadamente $20 \%{ }^{9}$. Sabe-se que o paciente obeso apresenta um sistema imune prejudicado, resultando em maior prevalência de infecções e úlceras de pressão, além de um tempo maior para cicatrização de feridas ${ }^{19}$.

Importante salientar o elevado percentual de pacientes sem registro de peso e/ou estatura no período de admissão na UTIP. Considera-se esta perda significativamente importante, podendo ter interferido nos resultados, seja na prevalência da desnutrição, assim como, na prevalência do excesso de peso. A ausência de dados antropométricos na admissão em uma unidade de terapia intensiva merece atenção da equipe multiprofissional, pois dificulta a avaliação nutricional e o cálculo das dosagens de medicações baseadas em peso corpóreo, além de comprometer o plano terapêutico nutricional, principalmente, quanto ao cálculo de macro e micronutrientes recomendadas pelas diretrizes de terapia nutricional para paciente crítico. Cabe ressaltar que, crescimento, um indicador de estado nutricional, e os índices antropométricos, são componentes essenciais de triagem nutricional e avaliação de pacientes pediátricos ${ }^{22}$. O peso é uma medida simples, mas por representar a soma de todos os compartimentos corporais, deve ser avaliado com cautela em algumas condições. É importante ressalvar que, a perda de peso, tem sido demonstrada como um indicador significativo de risco de mortalidade pós-operatório e de tempo de hospitalização ${ }^{23}$.

Crianças admitidas nas unidades de tratamento intensivo estão sob risco de alteração do estado nutricional e das medidas antropométricas ao longo dos dias. O déficit energético acumulado nos primeiros dias de internação nos pacientes gravemente doentes pode ter um papel importante nos resultados clínicos e no tempo de permanência nestas unidades ${ }^{24}$.

Portanto, na admissão de crianças e adolescentes na UTIP do Hospital de Clinicas de Porto Alegre, desnutrição foi o estado nutricional menos prevalente que eutrofia e excesso de peso, quando comparamos com outros 
estudos nacionais e internacionais, sendo que os casos de desnutrição não mostraram existir associação com doenças de base. Porém, déficit de estatura, comumente relacionado com desnutrição crônica, teve prevalência elevada e estatisticamente significativa, indicando a necessidade de mais estudos que esclareçam a associação deste déficit em população de crianças menores de dois anos em terapia intensiva. O número expressivo de prematuros encontrados no grupo constituído por menores de dois anos pode ter influenciado a prevalência de baixa estatura nesta população, independente do estado clínico. São necessários mais estudos sobre estado nutricional de pacientes críticos com história de prematuridade. Ainda, consideramos como principal limitação de nosso estudo a ausência de registro de peso e estatura em grande número de prontuários, o que pode ter contribuído na menor prevalência de desnutrição encontrada. Dessa forma, mais estudos são necessários, a fim de definir melhor o perfil clínico epidemiológico desta população pediátrica em estado crítico.

\section{REFERÊNCIAS}

1. Verger J. Nutrition in the pediatric population in the intensive care unit. Crit Care Nurs Clin North Am. 2014;26(2):199-215. https:// doi.org/10.1016/j.ccell.2014.02.005

2. Metha N, Bechard L, Cahill N. Nutritional practices and their relationship to clinical outcomes in critically ill children - An international multicenter cohort study. Crit Care Med. 2012;40(7): 2204-11. https://doi.org/10.1097/CCM.0b013e31824e18a8

3. Ong C, Han WM, Wong JJ, Lee JH. Nutrition biomarkers and clinical outcomes in critically ill children: A critical appraisal of the literature. Clin Nutr. 2014;33(2):191-7. https://doi.org/10.1016/j. clnu.2013.12.010

4. Powers J, Samaan K. Malnutrition in the ICU patient population. Crit Care Nurs Clin North Am. 2014;26(2):227-42 https://doi. org/10.1016/j.ccell.2014.01.003

5. Valla FV, Ford-Chessel C, Meyer R, Berthiller J, Dupenloup C, FollinArbelet N, Hubert A, Javouhey E, Peretti N. A training program for anthropometric measurements by a dedicated nutrition support team improves nutritional status assessment of the critically ill child. Pediatr Crit Care Med. 2015;16(3):e82-8. https://doi.org/10.1097/ PCC.0000000000000363

6. Green Corkins K. Nutrition-focused physical examination in pediatric patients. Nutr Clin Pract. 2015;30(2):203-9. https://doi. org/10.1177/0884533615572654

7. Cabral D, Velozo D, Piva J. Enfoque nutricional em unidade de terapia intensiva pediátrica. Sci Med. (Porto Alegre) 2013;23(2):127-33.

8. Zamberlan P, Delgado AF, Leone C, Feferbaum R, Okay TS. Nutrition therapy in a pediatric intensive care unit: indications, monitoring, and complications. JPEN J Parenter Enteral Nutr. 2011;35(4):523-9. https://doi.org/10.1177/0148607110386610

9. Goh VL, Wakeham MK, Brazauskas R, Mikhailov TA, Goday PS. Obesity is not associated with increased mortality and morbidity in critically ill children. JPEN J Parenter Enteral Nutr. 2013;37(1):102-8. https://doi.org/10.1177/0148607112441801
10. WHO. Multicentre Growth Reference Study Group. WHO Child Growth Standards: length/height-for-age, weight-for-age, weight-forlength, weight-for- height and body mass index-for-age: methods and development. Geneva: WHO; 2006.

11. Fenton TR, Kim JH. A Systematic review and meta-analysis to revise the Fenton Growth Chart for preterm infants. BMC Pediatr. 2013;13:59. https://doi.org/10.1186/1471-2431-13-59

12. Zamberlan P, Leone C, Tannuri U, Carvalho WB, Delgado AF. Nutritional risk and anthropometric evaluation in pediatric liver transplantation. Clinics (Sao Paulo). 2012;67(12):1387-92. https:// doi.org/10.6061/clinics/2012(12)07

13. Castro G, Kaufer-Horwitz M, Carrilo-López H A. Nutritional status of children in critical condition at admission to pediatric intensive care units. Bol Med Hosp Infant Mex. 2013;70(3):214-9.

14. Souza Menezes F, Leite HP, Koch Nogueira PC. Malnutrition as an independent predictor of clinical outcome in critically ill children. Nutrition. 2012;28(3):267-70. https://doi.org/10.1016/j. nut.2011.05.015

15. Sarni R, Carvalho MF, Monte C. Anthropometric evaluation, risk factors for malnutrition, and nutritional therapy for children in teaching hospitals in Brazil. J Pediatr (Rio J). 2009;85(3):223-8. https://doi.org/10.1590/S0021-75572009000300007

16. Cardoso-Demartini A, Bagatin A, Cavalcante da Silva R. Crescimento de crianças nascidas prematuras. Arq Bras Endocrinol Metab. 2011; 55(8):534-40. https://doi.org/10.1590/S0004-27302011000800006

17. Leroy JL, Ruel M, Habicht JP, Frongillo EA. Using height-for-age differences (HAD) instead of height-fork-age z-escores (HAZ) for the meaningful measurement of population-level catch up in linear growth in children less than 5 year of age. BMC Pediatr. 2015;15:145. https://doi.org/10.1186/s12887-015-0458-9

18. Damé P. Sistema de Vigilância Alimentar e Nutricional (SISVAN) em crianças do Rio Grande do Sul, Brasil: cobertura, estado nutricional e confiabilidade dos dados. Cad Saúde Pública. 2011;27(11): 2155-65. https://doi.org/10.1590/S0102-311X2011001100009

19. Martinez EE, Ariagno K, Arriola A, Lara K, Mehta NM. Challenges to nutrition therapy in the pediatric critically ill obese patient. Nutr Clin Pract. 2015;30(3):432-9. https://doi. org/10.1177/0884533615569887

20. Araújo CL, Dumith S, Menezes AM. Nutritional status of adolescents: the 11-year follow-up of the 1993 Pelotas (Brazil) birth cohort study. Cad Saúde Pública. 2010;26(10):1895-903. https://doi. org/10.1590/S0102-311X2010001000005

21. IBGE, Pesquisa Nacional de Saúde do Escolar [Internet]. Brasil. 2009 [capturado 2014 Out 10]. Disponível em: http//www.ibge.gov.br/ home/estatísticas/população/pense/Pense.pdf

22. ermilyea S, Slicker J, El-Chammas K, Sultan M, Dasgupta $M$, Hoffmann RG, Wakeham M, Goday PS. Subjective global nutritional assessment in critically ill children. JPEN J Parenter Enteral Nutr. 2013;37(5):659-66. https://doi.org/10.1177/0148607112452000

23. Sociedade Brasileira de Nutrição Parenteral e Enteral; Associação Brasileira de Nutrologia; Associação Médica Brasileira; Conselho Federal de Medicina. Projeto diretrizes. Triagem e avaliação do estado nutricional. São Paulo: AMB; 2011.

24. Marchetto da Silva F, Bermudes AC, Maneschy I. O impacto da introdução precoce de terapia nutricional enteral na redução da morbimortalidade na terapia intensiva pediátrica: uma revisão sistemática. Rev Assoc Med Bras. 2013;59(6):563-70. https://doi. org/10.1016/j.ramb.2013.06.013 